8 Rita Casetti, Chiara Agrati, Marianne Wallace, Sacchi A, Martini F, Martino A et al. Cutting edge: TGF- $\beta 1$ and IL-15 induce FOXP $3+\gamma \delta$ regulatory T cells in the presence of antigen stimulation. J Immunol 2009; 183: 3574-3577.

9 Li X, Kang N, Zhang X, Dong X, Wei W, Cui L et al. Generation of human regulatory gammadelta $T$ cells by TCRgammadelta stimulation in the presence of TGF-beta and their involvement in the pathogenesis of systemic lupus erythematosus. $J$ Immunol 2011; 186: 6693-6700.

10 Lal G, Zhang N, van der Touw W, Ding Y, Ju W, Bottinger EP et al. Epigenetic regulation of Foxp3 expression in regulatory $T$ cells by DNA methylation. J Immunol 2009; 182: 259-273.

11 Choi J, Ritchey J, Prior JL, Holt M, Shannon WD, Deych E et al. In vivo administration of hypomethylating agents mitigate graft-versus-host disease without sacrificing graft-versus-leukemia. Blood 2010; 116: 129-139.
12 Beres A, Komorowski R, Mihara M, Drobyski WR. Instability of Foxp3 expression limits the ability of induced regulatory $T$ cells to mitigate graft versus host disease. Clin Cancer Res 2011; 17: 3969-3983.

13 Dander E, Lucchini G, Vinci P, Introna M, Masciocchi F, Perseghin P et al. Mesenchymal stromal cells for the treatment of graft-versus-host disease: understanding the in vivo biological effect through patient immune monitoring. Leukemia 2012; 26: 1681-1684.

14 Plumas J, Manches O, Chaperot L. Mechanisms of action of extracorporeal photochemotherapy in the control of GVHD: involvement of dendritic cells. Leukemia 2003; 17: 2061-2062.

15 Hippen KL, Riley JL, June CH, Blazar BR. Clinical perspectives for regulatory T cells in transplantation tolerance. Semin Immunol 2011; 23: 462-468.

\title{
OPEN
}

\section{Deficiency of CREB and over expression of miR-183 in juvenile myelomonocytic leukemia}

\author{
Leukemia (2013) 27, 1585-1588; doi:10.1038/leu.2013.49
}

Juvenile myelomonocytic leukemia (JMML) is a rare leukemia of infancy and early childhood. A hallmark of JMML is that JMML cells are selectively hypersensitive to GM-CSF in vitro, but have normal sensitivity to IL-3, which shares the $\beta$-subunit receptor with GM-CSF. ${ }^{1,2}$ The pathogenesis of JMML is linked to dysregulated signal transduction through the Ras signaling pathway caused by mutations of RAS, PTPN11 and c-CBL, or loss-of-heterozygosity of NF1., Although most patients with JMML experience an aggressive disease course, some others spontaneously resolve., No specific mutation has been definitively demonstrated to confer a poorer prognosis, with the exception of perhaps PTPN11, which has been shown to be associated with a higher risk of relapse in some series. In addition, gene expression and methylation analyses have identified signatures associated with outcome that are unexpectedly independent of genotype, ${ }^{5,6}$ leading us to believe that additional mutations or events mitigate the course of JMML. Finally, the mechanisms that underlie selective GM-CSF hypersensitivity are not well established in JMML.

We previously reported that constitutively-hyperactive MAPK and Akt were found in $73 \%$ and $55 \%$ of JMML patients, respectively, and that a tumor suppressor, PTEN (phosphatase and tensin homolog deleted on chromosome ten), was deficient in $67 \%$ of JMML patients. ${ }^{7}$ As PTEN functions as an antagonist to MAPK and PI3K/Akt activities, ${ }^{7}$ its deficiency in JMML might therefore result in an inability to antagonize the over-activity demonstrated by GM-CSF hypersensitivity. However, PTEN has never been linked to GM-CSF signaling specifically. Therefore, in this study, we sought to identify additional molecules that specifically affect GM-CSF signaling in JMML. One such possible protein is CREB (CAMP-response-element-binding protein), which is a member of the activating transcription factor family. The phosphorylation status of CREB determines whether it acts on the c-Jun promoter in a repressive or activating manner to affect c-Jun gene transcription. ${ }^{8}$ c-Jun has been found to be overexpressed in JMML, which is a coactivator of PU.1 in promoting monocytic differentiation. ${ }^{9}$ CREB has also been described as a protooncogene, overexpressed in $65 \%$ of acute myeloid leukemia (AML), and is related to growth dynamics for colony-forming unit/ granulocyte macrophage (CFU-GM). ${ }^{10}$
In order to test our hypothesis that there might be a CREBrelated aberrancy in JMML, we collected peripheral blood (PB) or bone marrow (BM) from $47 \mathrm{JMML}$ patients at diagnosis, and evaluated CREB protein levels in mononuclear cells (MNCs) from 26 of these who had sufficient sample material for western blot. In contrast to the data reported in AML, we found that 22/26 (85\%) of subjects had substantially lower CREB protein levels than normal controls (Figure 1a). In further quantifying the total CREB protein using ELISA, we found that the median level of CREB protein was significantly lower in JMML subjects $(0.62 \mathrm{ng} / \mathrm{mg} \mathrm{BSA}$, $n=26)$ as compared with 7 normal controls $(8.8 \mathrm{ng} / \mathrm{mg}$ BSA, $P=0.006$; Figure $1 \mathrm{~b}$ ). To understand the mechanism underlying CREB deficiency in JMML, we evaluated the mRNA expression of $C R E B$ in MNCs from $31 \mathrm{JMML}$ patients using relative quantitative real-time RT-PCR (qRT-PCR). The median of the relative quantity (RQ) of CREB mRNA from patients was significantly lower than that from 17 normal adults ( 0.42 vs $1.00, P<0.001$, Figure $1 c)$. This indicates that the regulation of $C R E B$ transcription is disrupted in JMML. However, there was no linear correlation between CREB mRNA and CREB protein levels in JMML, suggesting that additional mechanism(s) affecting protein levels may be involved. These results, together with the AML data, ${ }^{10}$ suggest that $a$ balanced amount of CREB is critical for maintaining cells with proper responsiveness to GM-CSF in hematopoiesis.

Phosphorylation at Ser-133 of CREB is required for the transcriptional activation of early growth response gene-1 (Egr-1) in response to GM-CSF stimulation in hematopoietic cell lines, but not to IL-3 stimulation. ${ }^{11}$ Egr-1 upregulates the PTEN gene. Mice lacking Egr-1 exhibit a significant increase in steady-state levels of dividing hematopoietic stem cells (HSCs) in the BM, and a striking spontaneous mobilization of HSCs into blood. ${ }^{12}$ Egr-1 has a deterministic role in governing the development of hematopoietic cells along the macrophage lineage. ${ }^{13}$ Therefore, we hypothesized that Egr-1 expression may be deficient in JMML because of the deficient transcriptional activation of CREB. We first evaluated Egr-1 protein levels by western blot in MNCs of 24 patients. We found that $21 / 24(87 \%)$ of subjects were substantially Egr-1 protein deficient (Figure 1a). If additional studies confirm our data, Egr-1 would be the most frequent protein deficiency in JMML. Owing to no commercial ELISA assay being available for Egr-1 protein quantification, we were unable to quantitatively evaluate the levels of Egr-1 protein in JMML. Instead, we quantified Egr-1 
a

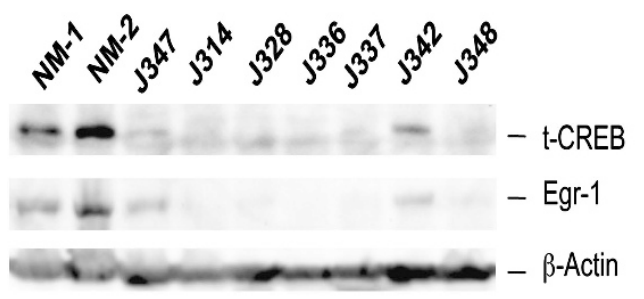

C

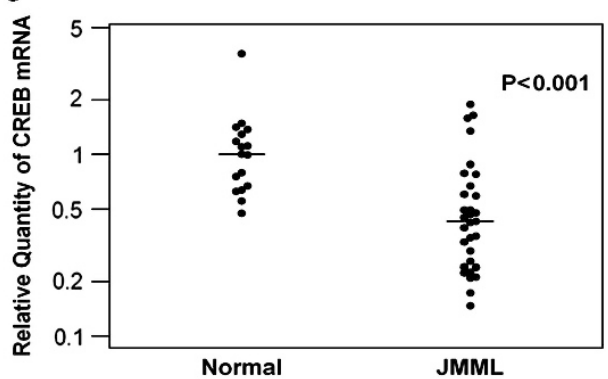

d
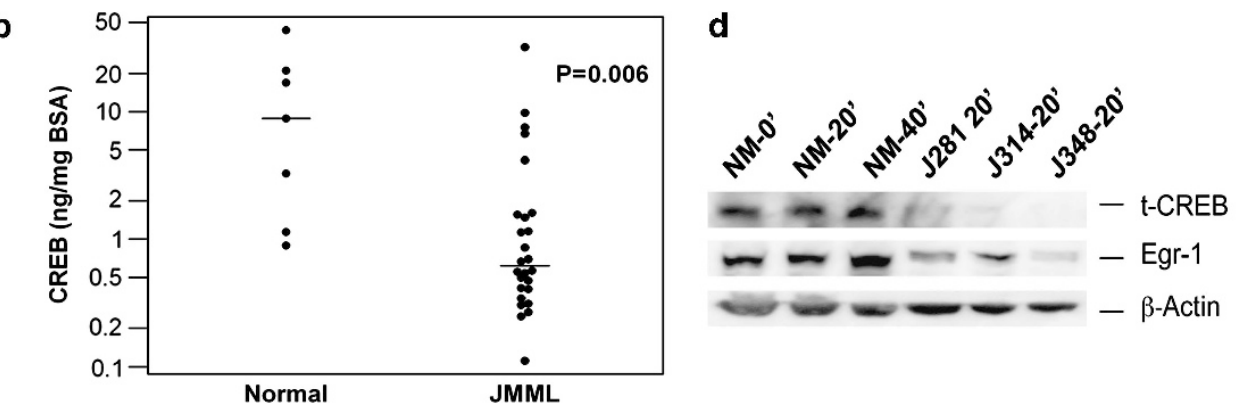

Figure 1. (a) Total CREB and Egr-1 protein levels in JMML. Representative lysates of unstimulated MNCs were prepared from PB or BM. CREB, Egr-1 and $\beta$-actin proteins were analyzed by western blot. NM, normal individual; J\#, JMML patient. (b) Total CREB protein levels were quantified using ELISA kit. Dots show the CREB protein levels in normal controls or JMML patients. The bars indicate the median levels of each group (8.8 vs $0.6, P=0.006$ ). Normal, $n=7 ;$ JMML, $n=26$. (c) CREB mRNA expression level of MNCs was quantified by real-time qRT-PCR. Total RNA was extracted from unstimulated MNCs from PB or BM of patients or normal controls. Dots show the CREB expression levels in normal controls or JMML patients. The bars indicate the median levels of each group (1.0 vs $0.4, P<0.001)$. Normal controls, $n=17$; JMML patients, $n=31$. (d) Total CREB and Egr-1 protein levels in CFU-GM cells from JMML patients and a normal control. Frozen MNCs (10 ${ }^{7} / \mathrm{sample}$ ) from patients or normal control were plated in a density of $10^{5} / \mathrm{ml}$ on methylcellulose-based medium containing rhGM-CSF $2 \mathrm{ng} / \mathrm{ml}$. CFU-GM cells were collected after incubation for 14 days, and washed in PBS twice, then starved in RPMI-1640 with $10 \%$ FBS without rhGM-CSF for $16 \mathrm{~h}$. Cells were then stimulated with rhGM-CSF at a concentration of $10 \mathrm{ng} / \mathrm{ml}$ in RPMI-1640 with 10\% FBS. Cells were collected and washed twice in cold PBS at the proper time-points, and cell lysates were prepared. Protein levels were analyzed by western blot.

mRNA expression in MNCs from 47 JMML patients using qRT-PCR. We found that Egr-1 expression was only slightly lower in JMML (median 4.30, $n=47$ ) than in normal controls (median 4.66, $n=17$, $P>0.05)$. This indicated that the Egr-1 transcriptional activity was not significantly disrupted in JMML, suggesting a post-transcriptional mechanism is responsible for the decreased Egr-1 protein levels in JMML.

In order to exclude that the decreased expressions of CREB and Egr-1 were caused by sample degradation, we collected cells from expanded CFU-GM derived from MNCs of a normal control $\mathrm{BM}$ and three JMML patients. We found that in the presence of $10 \%$ fetal bovine serum and rhGM-CSF, both CREB and Egr-1 were deficient in fresh JMML CFU-GM in comparison with the normal control (Figure 1d). This confirms that JMML cells are truly deficient with both CREB and Egr-1.

Mutations are rarely reported in either CREB or Egr-1 genes in patients with leukemia or other forms of cancer. Pigazzi et al. ${ }^{14}$ reported that CREB was overexpressed in AML owing to the hypermethylated promoter of miR-34b. Further, knockdown of miR-183 increased Egr-1 and PTEN expression in cancer cells. ${ }^{15}$ Interestingly, miR-34b is located on chromosome 11q23, and miR-183 on $7 q 32$. Both of these chromosomal segments are frequently disrupted in JMML., ${ }^{3,6}$ Therefore, we evaluated the expression levels of miR-34b and miR-183 in MNCs from PB or BM of $47 \mathrm{JMML}$ patients. We found a slightly higher median level of miR-34b in JMML subjects (median $=1.4$ vs $1.0, P>0.05$, Figure 2a). Strikingly, the median level of miR-183 was significantly higher in JMML compared with normal controls (median $=13.8$ vs 4.2, $P<0.001$, Figure 2b). Our data suggest that miR-183 may contribute to Egr-1 protein deficiency in JMML, but miR-34b does not have a significant role in CREB deficiency. Interestingly, we also found a significant linear correlation between the expression level of miR-183 and the monocyte percentage in 34 patients who had available clinical data $(P=0.026$, Figure $2 \mathrm{c})$. On the basis of a robust regression analysis, for every unit increased in the square root of RQ miR-183, the monocyte percentage significantly increased by $0.73 \%$ (s.e. $=0.32 \%, P<0.05$ ). Unfortunately at present, we do not have access to clinical data to analyze the correlation of miR-183 expression levels with patients' clinical course. However, in further investigating miR-183, attempting a correlation with the known mutational status of PTPN11, NRAS, KRAS and CBL genes in 28 patients with available data (Supplementary Table-S1), we found that miR-183 expression was significantly higher in patients with PTPN11 mutations (median 34.8) than those without (median 6.9, $P=0.002$, Figure $2 \mathrm{~d}$ ). We could not make conclusive correlations between miR-183 expression and NRAS, KRAS and CBL mutational status because of limited numbers of patients with these mutations.

In this study, we found that CREB was significantly decreased in JMML, and this decrease may contribute to overexpression of C-Jun in JMML, ${ }^{9}$ because of insufficient CREB repression of transcriptional activation on C-Jun. ${ }^{8}$ We also found a significant overexpression of miR-183 in JMML, which may contribute to Egr-1 protein deficiency, and further worsen the monocyte accumulation in JMML. This is supported by our finding that the expression levels of miR-183 were significantly linearly correlated with the monocyte percentage in JMML patients. Furthermore, it has been reported that anti-miR-183 treatment leads to increased protein levels of Egr-1 and PTEN in tumor cell lines. ${ }^{15}$ Therefore, miR-183 may be a potential biomarker and therapeutic target for JMML. The mechanism of overexpression of miR-183 is unknown in JMML. We found that overexpression of miR-183 was related to PTPN11 mutation. Yang et al. ${ }^{9}$ reported that activating PTPN11 mutants increased c-Jun-PU.1 complexes and promoted 

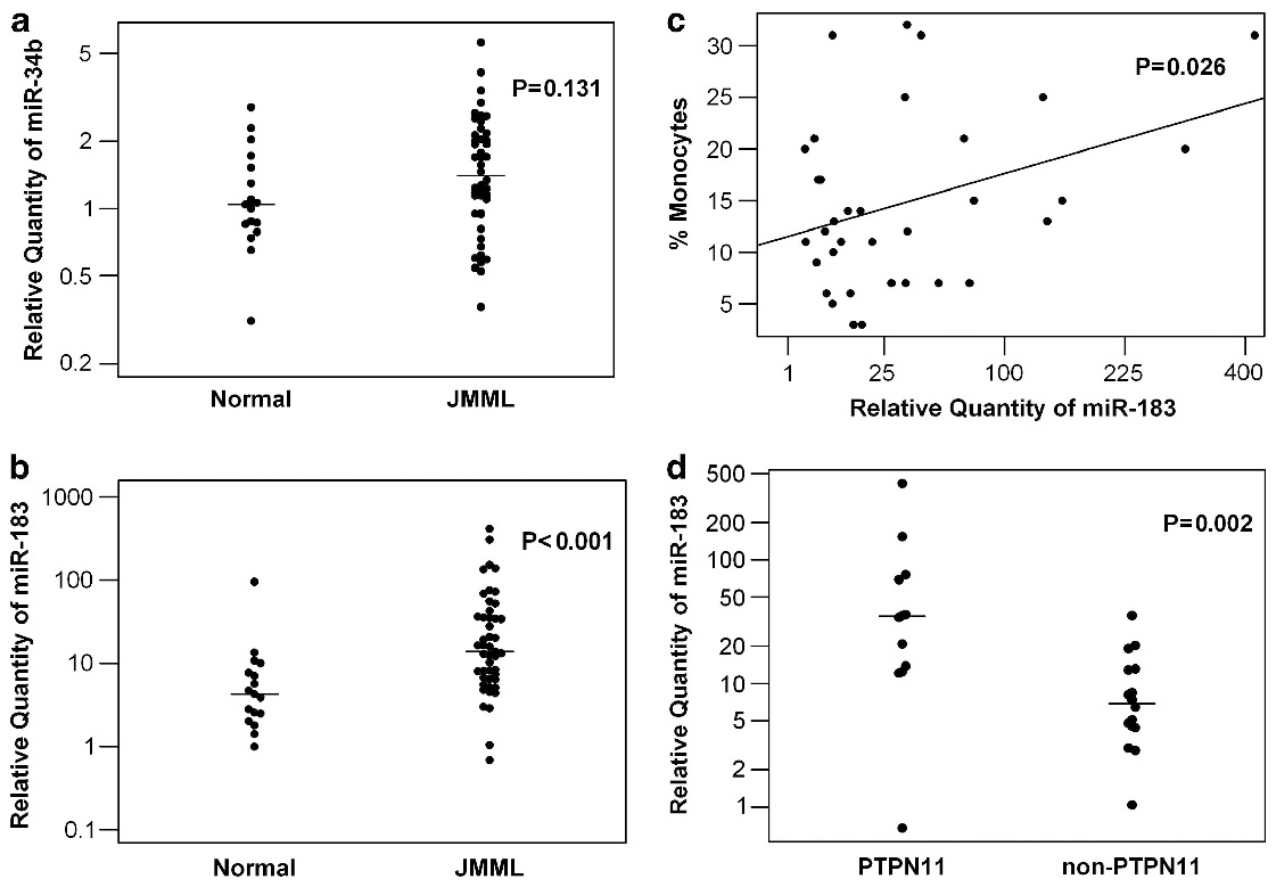

Figure 2. (a) miR-34b expression levels of MNCs were quantified by real-time qRT-PCR. Total RNA was extracted from unstimulated MNCs from $\mathrm{PB}$ or BM of patients or normal controls. Dots show the miR-34b expression levels in normal controls $(n=17)$ or JMML patients $(n=47)$. The bars indicate the median levels of each group. (b) miR-183 expression levels of MNCs were quantified by real-time qRT-PCR as miR-34b. Dots show the miR-183 expression levels in normal controls $(n=17)$ or JMML patients $(n=47)$. The bars indicate the median levels of each group. (c) Linear correlation between miR-183 expression levels in MNCs and the monocyte percentages in PB of JMML patients $(n=34, P<0.05)$. (d) miR-183 expression levels of MNCs in patients with or without PTPN11 mutation. Dots show the miR-183 expression levels in patients with PTPN11 mutation $(n=12)$ or without $(n=16)$. The bars indicate the median levels of each group.

monocytic differentiation. It will be interesting to investigate whether increased c-Jun-PU.1 complexes have any role in upregulating miR-183 expression, or overexpression of miR-183 is correlated with any status of chromosome $7 q$ in JMML.

In summary, we identified CREB and Egr-1 as being significantly deficient in JMML, both of which are molecules specifically affecting GM-CSF signaling. We also demonstrated that miR-183 was overexpressed in JMML, which may contribute to the Egr-1 deficiency and possibly the monocyte predominance in JMML. This is the first evidence demonstrating that a microRNA is involved in JMML pathogenesis. Further investigations may reveal how miR-183 is regulated and contributes to dysregulated GM-CSF signaling, and provide more insights to microRNA involvement in the pathogenesis of other forms of leukemia.

\section{CONFLICT OF INTEREST}

The authors declare no conflict of interest.

\section{ACKNOWLEDGEMENTS}

This research was supported by the United States of America National Institutes of Health grants UL1 TR000039, R01 CA095621 and R01 HL082959 (PDE and YLL). We thank Priyangi A. Malaviarachchi for expert technical assistance.

\section{NOTE ADDED IN PROOF}

While this manuscript was undergoing revision, a manuscript was published by Pigazzi et al, Haematologica, 2012 Oct 25 (published online ahead of print) 'miR-34b promoter hypermethylation induces CREB overexpression and contributes to myeloid transformation.' In this manuscript, the authors present some data from JMML patient samples to suggest CREB protein deficiency.

\section{YL Liu', SY Lensing ${ }^{1}$, Y Yan ${ }^{1}$, TM Cooper ${ }^{2}$, ML Loh ${ }^{3}$ and PD Emanuel ${ }^{1}$}

${ }^{1}$ Department of Medicine, Winthrop P Rockefeller Cancer Institute, University of Arkansas for Medical Sciences, Little Rock, AR, USA;

${ }^{2}$ Department of Pediatrics, Division of Hematology/Oncology, Emory University, Atlanta, GA, USA and

${ }^{3}$ Division of Hematology Oncology, University of California Benioff Children's Hospital, San Francisco, CA, USA E-mail: ylucyliu@uams.edu or pdemanuel@uams.edu

\section{REFERENCES}

1 Kitamura T, Sato N, Arai K, Miyajima A. Expression cloning of the human IL-3 receptor CDNA reveals a shared beta subunit for the human IL-3 and GM-CSF receptors. Cell 1991; 66: 1165-1174.

2 Emanuel PD, Bates L, Castleberry RP, Gualtieri RJ, Zuckerman KS. Selective hypersensitivity to granulocyte-macrophage colony-stimulating factor by juvenile chronic myeloid leukemia hematopoietic progenitors. Blood 1991; 77: 925-929.

3 Chan RJ, Cooper T, Kratz CP, Weiss B, Loh ML. Juvenile myelomonocytic leukemia: a report from the 2nd International JMML Symposium. Leuk Res 2009; 33: 355-362.

4 Loh ML, Sakai DS, Flotho C, Kang M, Fliegauf M, Archambeault S et al. Mutations in CBL occur frequently in juvenile myelomonocytic leukemia. Blood 2009; 114: 1859-1863.

5 Olk-Batz C, Poetsch AR, Nollke P, Claus R, Zucknick M, Sandrock I et al. Aberrant DNA methylation characterizes juvenile myelomonocytic leukemia with poor outcome. Blood 2011; 117: 4871-4880.

6 Bresolin S, Zecca M, Flotho C, Trentin L, Zangrando A, Sainati L et al. Gene expression-based classification as an independent predictor of clinical outcome in juvenile myelomonocytic leukemia. J Clin Oncol 2010; 28: 1919-1927.

7 Liu YL, Castleberry RP, Emanuel PD. PTEN deficiency is a common defect in juvenile myelomonocytic leukemia. Leuk Res 2009; 33: 671-677.

8 Lamph WW, Dwarki VJ, Ofir R, Montminy M, Verma IM. Negative and positive regulation by transcription factor CAMP response element-binding protein is modulated by phosphorylation. Proc Natl Acad Sci USA 1990; 87: 4320-4324. 
9 Yang Z, Kondo T, Voorhorst CS, Nabinger SC, Ndong L, Yin F et al. Increased c-Jun expression and reduced GATA2 expression promote aberrant monocytic differentiation induced by activating PTPN11 mutants. Mol Cell Biol 2009; 29: 4376-4393.

10 Cheng JC, Kinjo K, Judelson DR, Chang J, Wu WS, Schmid I et al. CREB is a critical regulator of normal hematopoiesis and leukemogenesis. Blood 2008; 111 $1182-1192$.

11 Lee HJ, Mignacca RC, Sakamoto KM. Transcriptional activation of egr-1 by granulocyte-macrophage colony-stimulating factor but not interleukin 3 requires phosphorylation of cAMP response element-binding protein (CREB) on serine 133 J Biol Chem 1995; 270: 15979-15983.

12 Min IM, Pietramaggiori G, Kim FS, Passegue E, Stevenson KE, Wagers AJ. The transcription factor EGR1 controls both the proliferation and localization of hematopoietic stem cells. Cell Stem Cell 2008; 2: 380-391.
13 Krishnaraju K, Hoffman B, Liebermann DA. Early growth response gene 1 stimulates development of hematopoietic progenitor cells along the macrophage lineage at the expense of the granulocyte and erythroid lineages. Blood 2001; 97: 1298-1305.

14 Pigazzi M, Manara E, Baron E, Basso G. miR-34b targets cyclic AMP-responsive element binding protein in acute myeloid leukemia. Cancer Res 2009; 69: 2471-2478

15 Sarver AL, Li L, Subramanian S. MicroRNA miR-183 Functions as an Oncogene by Targeting the Transcription Factor EGR1 and Promoting Tumor Cell Migration. Cancer Res 2010; 70: 9570-9580.

(c) (i) $\Theta$ This work is licensed under a Creative Commons AttributionNonCommercial-NoDerivs 3.0 Unported License. To view a copy of this license, visit http://creativecommons.org/licenses/by-nc-nd/3.0/

\section{$G A D D 45 A$ methylation predicts poor overall survival in acute myeloid leukemia and is associated with $I D H 1 / 2$ and $D N M T 3 A$ mutations}

\section{Leukemia (2013) 27 1588-1592; doi:10.1038/leu.2012.346}

Acute myeloid leukemia (AML) is a heterogeneous disease with variable treatment outcome. Despite recent advances in understanding the key molecular mechanisms of myeloid leukemogenesis, risk stratification remains imperfect, particularly in the intermediate risk group in which a large proportion of patients have a normal karyotype. For this group of patients, genetic alterations in genes, such as FLT3, NPM1 and CEPBA, provide useful prognostic information, but alone are not sufficient to accurately predict prognosis for all patients. Recently, recurrent mutations in epigenetic modifiers such as IDH1/2, ${ }^{1}$ DNMT3A, ${ }^{2}$ and $T E T 2^{3}$ have been described, but their prognostic significance remains somewhat controversial, and it is unclear if targeted therapies will evolve from identification of these mutations. ${ }^{4}$ Identification of target genes that undergo epigenetic modification in the landscape of somatic mutations in epigenetic modifiers is likely to yield important prognostic information, and potentially identify novel biomarkers of response to demethylating agents, which are currently showing promise in the treatment of AML.

Growth arrest and DNA-damage inducible alpha (GADD45A) is a tumour suppressor gene that has cell-type specific roles in cellular stress, coordinating DNA repair and de-methylation, cell cycle arrest and pro-apoptotic or pro-survival responses., ${ }^{5,6}$ Although downregulation of GADD45A has been linked to the presence of FLT3-ITD and FLT3-TKD mutants, ${ }^{7}$ and recently with RUNX1 c-terminal mutations, ${ }^{8}$ there is a broad downregulation of GADD45A in AML and other mechanisms associated with this remain to be determined. Previous studies in solid tumours have identified atypical methylation of GADD45A at four discrete CpG residues (CpG1-4) $\sim 700 \mathrm{bp}$ upstream of the transcriptional start site, which correlates with gene expression and has been linked to tumour response in vitro and in xenograft models. ${ }^{7,9-11}$ Here, we assessed DNA methylation of these same key CpG residues in GADD45A in AML and determined co-occurrence with other recurrent mutations and impact on patient outcome.

To determine the frequency of GADD45A CpG1-4 methylation in $A M L$, we screened a retrospective cohort of 222 newly diagnosed AML cases for methylation of GADD45A CpG1-4 using Sequenom
MasSARRAY on bisulphite converted genomic DNA (amplicon given in Figure 1a). All patients were collected with informed consent with approval from the relevant ethics committees, in accordance with the Declaration of Helsinki. The levels of GADD45A CpG1-4 methylation in the patient samples ranged from $0-90 \%$ (Figure 1b). As CpG1 methylation was always higher than CpG2-4 methylation it was considered representative of methylation at this discrete region, and GADD45A CpG1 hypermethylation (G45Ame ${ }^{\text {high }}$ ) was defined as methylation exceeding 2 s.d. from the mean of the eight normal bone marrow and three normal $\mathrm{CD}_{3}{ }^{+}$pooled controls ( $\geqslant 12 \%$; Figure $1 \mathrm{~b}$ ). Using this cutoff, 93 of 222 AML patients (42\%) were defined as G45Ame high $^{\text {hish }}$ (Figure 1b; Table 1). G45Ame ${ }^{\text {high }}$ patients were significantly older than $645 A$ me ${ }^{\text {low }}$ patients with a mean age of 60 vs 55 years $(P=0.02$; Table 1$)$. There was also a sex bias with significantly more females than males in the G45Ame high group. Additionally,

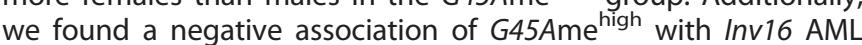
$(P=0.006$; Table 1$)$.

To assess co-occurrence with other common mutations in AML we used a PCR-based fragment analysis for FLT3-ITD screening and a multiplexed matrix-assisted laser desorption/ionization time-offlight genotyping approach (Sequenom MassARRAY Compact System, Sequenom, Inc., San Diego, CA, USA) for detection of the following mutations: KIT (D816V), DNMT3A (R882C/H), FLT3 (TKD: $\mathrm{D} 835 \mathrm{H} / \mathrm{Y} / \mathrm{N} / \mathrm{E}$, I836DEL, I836INS), IDH1 (R132C/H/P), IDH2 (R140W/ L/G, R172W/G/K/M), JAK1 (T478S, V623A), JAK2 (V617F), KRAS (G12D/N/A, G13D/A) and WT1 (rs16754). Mutation frequencies are given in Table 1 . We subsequently assessed co-association of recurrent mutations with GADD45A methylation status. Interestingly, there was a significant association of G45Ame high with IDH1/ $2(P<0.0001)$ and DNMT3A $(P=0.02)$ mutations (Table 1, Figure 1c). IDH1 and IDH2 mutations were mutually exclusive as reported previously, ${ }^{1}$ and 36 of 41 patients $(88 \%, P<0.0001)$ with either IDH1 or IDH2 mutations were in the G45Ame ${ }^{\text {high }}$ patient group (Figure 1c). For DNMT3A, 19 of 30 patients were associated with the G45Ame high patient group (63\%, $P=0.02$ ). We found no statistical association of G45Ame high with FLT3-ITD or other recurrent mutations, and 13 of 93 (14\%) G45Ame ${ }^{\text {high }}$ patients were negative for all mutations tested. 